\title{
On the Sixth Power Mean Value of the Generalized Three-Term Exponential Sums
}

\author{
Yahui Yu ${ }^{1}$ and Wenpeng Zhang ${ }^{2}$ \\ ${ }^{1}$ Luoyang Institute of Science and Technology, Luoyang, Henan 471023, China \\ ${ }^{2}$ School of Mathematics, Northwest University, Xi'an, Shaanxi 710127, China \\ Correspondence should be addressed to Wenpeng Zhang; wpzhang@nwu.edu.cn
}

Received 20 January 2014; Accepted 18 March 2014; Published 9 April 2014

Academic Editor: Shanhe Wu

Copyright (C) 2014 Y. Yu and W. Zhang. This is an open access article distributed under the Creative Commons Attribution License, which permits unrestricted use, distribution, and reproduction in any medium, provided the original work is properly cited.

The main purpose of this paper is using the estimate for trigonometric sums and the properties of the congruence equations to study the computational problem of one kind sixth power mean value of the generalized three-term exponential sums and give an exact computational formula for it.

\section{Introduction}

Let $q \geq 3$ be a positive integer and let $\chi$ be any Dirichlet character $\bmod q$. For any integers $m$ and $n$, the generalized three-term exponential sum $C(m, n, k, \chi ; q)$ is defined as follows:

$$
C(m, n, k, \chi ; q)=\sum_{a=1}^{q-1} \chi(a) e\left(\frac{a^{k}+m a^{2}+n a}{q}\right),
$$

where $k \geq 3$ is a fixed integer and $e(y)=e^{2 \pi i y}$.

Many authors have studied this and related exponential sums and obtained a series of results; some related contents can be found in [1-9]. For example, Du and Han [5] proved that, for any integer $k \geq 3$, we have the identity

$$
\sum_{m=1}^{p} \sum_{n=1}^{p}\left|\sum_{a=1}^{p-1} e\left(\frac{m a^{k}+n a^{2}+a}{p}\right)\right|^{4}=2 p^{4}-3 p^{3}-p^{2} \cdot C(k, p),
$$

where the constant $C(k, p)$ is defined as follows:

$$
C(k, p)=\sum_{\substack{a=1 \\ a^{k}+b^{k} \equiv c^{k}+1 \bmod p \\ a^{2}+b^{2} \equiv c^{2}+1 \bmod p}}^{p-1 p-1 p-1} 1
$$

In particular, if $k=6$, then we have the identity

$$
\begin{aligned}
\sum_{m=1}^{p} \sum_{n=1}^{p} \mid & \left|\sum_{a=1}^{p-1} e\left(\frac{m a^{6}+n a^{2}+a}{p}\right)\right|^{4} \\
& = \begin{cases}2 p^{4}-11 p^{3}+16 p^{2}, & \text { if } p \equiv 3 \bmod 4 \\
2 p^{4}-15 p^{3}+36 p^{2}, & \text { if } p \equiv 1 \bmod 4 .\end{cases}
\end{aligned}
$$

It seems that no one has studied the sixth power mean of the generalized three-term exponential sums

$$
\sum_{m=1}^{p} \sum_{n=1}^{p} \sum_{\chi \bmod }\left|\sum_{a=1}^{p-1} \chi(a) e\left(\frac{a^{k}+m a^{2}+n a}{p}\right)\right|^{6}
$$

where $\chi \bmod p$ denotes the summation over all characters $\chi \bmod p$. The problem is interesting, because it can reflect more or less the upper bound estimates of $C(m, n, k, \chi ; p)$. It is easy to see that mean value (2) is the best possible. So, we have reason to believe that (5) and (2) have similar asymptotic properties. In fact, we can use the analytic method and the properties of the congruence equation to give an exact computational formula for (5). That is, we will prove the following. 
Theorem 1. Let $p>3$ be a prime. Then, for any integer $k \geq 3$ with $(k, p-1)=1$, we have the identity

$$
\begin{gathered}
\sum_{m=1}^{p} \sum_{n=1}^{p} \sum_{\chi \bmod p}\left|\sum_{a=1}^{p-1} \chi(a) e\left(\frac{a^{k}+m a^{2}+n a}{p}\right)\right|^{6} \\
=p^{2} \cdot(p-1)^{2} \cdot\left(6 p^{2}-21 p+19\right) .
\end{gathered}
$$

It is very strange that the mean value in our theorem is independent of the size of $k$; it depends only on whether $(k, p-1)=1$ or $(k, p-1)>1$. For any Dirichlet character $\chi$ mod $p$, whether there exists a computational formula for the sixth power mean value

$$
\sum_{m=1}^{p} \sum_{n=1}^{p}\left|\sum_{a=1}^{p-1} \chi(a) e\left(\frac{a^{k}+m a^{2}+n a}{p}\right)\right|^{6}
$$

or $2 h$-th $(h \geq 4)$ power mean value

$$
\sum_{m=1}^{p} \sum_{n=1}^{p} \sum_{\chi \bmod }\left|\sum_{a=1}^{p-1} \chi(a) e\left(\frac{a^{k}+m a^{2}+n a}{p}\right)\right|^{2 h}
$$

is two open problems, which we will further study.

\section{Proof of Theorem 1}

In this section, we will give the proof of our theorem directly. Hereinafter, we will use many properties of trigonometric sums and congruence equation, all of which can be found in $[6,10]$, so they will not be repeated here. Note that $(k, p-1)=$ 1 , from the trigonometric identity

$$
\sum_{a=0}^{p-1} e\left(\frac{n a}{p}\right)= \begin{cases}p, & \text { if }(n, p)=p \\ 0, & \text { if }(n, p)=1\end{cases}
$$

Regarding the properties of reduced residue system mod $p$ and the orthogonality relation for characters $\bmod p$, we have

$$
\begin{aligned}
& \sum_{m=1}^{p} \sum_{n=1}^{p} \sum_{\bmod p}\left|\sum_{a=1}^{p-1} \chi(a) e\left(\frac{a^{k}+m a^{2}+n a}{p}\right)\right|^{6} \\
& =\sum_{a=1}^{p-1} \sum_{b=1}^{p-1} \sum_{c=1}^{p} \sum_{d=1}^{p} \sum_{e=1}^{p-1} \sum_{f=1}^{p-1} \sum_{\chi} \chi(a b c \bar{d} \bar{e} \bar{e} \bar{f}) e\left(\frac{a^{k}+b^{k}+c^{k}-d^{k}-e^{k}-f^{k}}{p}\right) \\
& \times \sum_{m=1}^{p} \sum_{n=1}^{p} e\left(\frac{m\left(a^{2}+b^{2}+c^{2}-d^{2}-e^{2}-f^{2}\right)+n(a+b+c-d-e-f)}{p}\right) \\
& =\sum_{a=1}^{p-1} \sum_{b=1}^{p-1} \sum_{c=1}^{p-1} \sum_{d=1}^{p-1} \sum_{e=1}^{p-1} \sum_{f=1}^{p-1} \sum_{\chi} \chi(a b c \bar{d} \bar{e} \bar{e}) e\left(\frac{f^{k}\left(a^{k}+b^{k}+c^{k}-d^{k}-e^{k}-1\right)}{p}\right) \\
& \times \sum_{m=1}^{p} \sum_{n=1}^{p} e\left(\frac{m f^{2}\left(a^{2}+b^{2}+c^{2}-d^{2}-e^{2}-1\right)+n f(a+b+c-d-e-1)}{p}\right) \\
& =p^{2} \sum_{\substack{a=1 \\
a+b+c \equiv d+e+1 \bmod p \\
a^{2}+b^{2}+c^{2} \equiv d^{2}+e^{2}+1 \bmod p}}^{p-1 p-1 p-1 p-1 p-1} \sum_{\substack{c=1 \\
a^{2}}} \chi(a b c \bar{d} \bar{e}) \sum_{f=1}^{p-1} e\left(\frac{f\left(a^{k}+b^{k}+c^{k}-d^{k}-e^{k}-1\right)}{p}\right)
\end{aligned}
$$

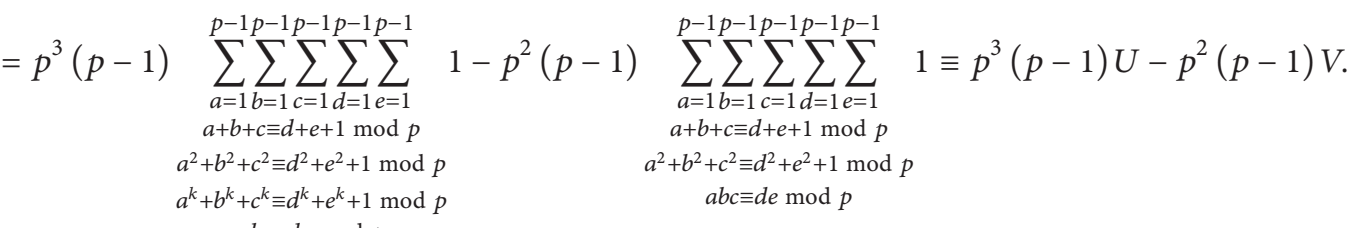

Now, we compute the values of $U$ and $V$ in (10), respectively. It is clear that the value of $V$ is equal to the number of the solutions of the system of the congruence equations:

$$
a+b+c \equiv d+e+1 \bmod p,
$$

$$
\begin{aligned}
a^{2}+b^{2}+c^{2} & \equiv d^{2}+e^{2}+1 \bmod p \\
a b c & \equiv d e \bmod p
\end{aligned}
$$

where $1 \leq a, b, c, d, e \leq p-1$. 
The system of congruence equation (11) is equivalent to the system of the congruence equations:

$$
\begin{gathered}
a+b+c \equiv d+e+1 \bmod p, \\
a b+b c+c a \equiv d e+d+e \bmod p, \\
a^{2}+b^{2}+c^{2} \equiv d^{2}+e^{2}+1 \bmod p, \\
a b c \equiv d e \bmod p .
\end{gathered}
$$

That is equivalent to the system of the congruence equations as follows:

$$
\begin{gathered}
a b c+a+b+c-1 \equiv a b+b c+c a \bmod p, \\
a+b+c \equiv d+e+1 \bmod p \\
a^{2}+b^{2}+c^{2} \equiv d^{2}+e^{2}+1 \bmod p \\
(a-1)(b-1)(c-1) \equiv 0 \bmod p \\
a+b+c \equiv d+e+1 \bmod p \\
a^{2}+b^{2}+c^{2} \equiv d^{2}+e^{2}+1 \bmod p
\end{gathered}
$$

For all integers $1 \leq a, b, c, d, e \leq p-1$, we compute the number of the solutions of (14). We separate the solutions of (14) into three cases as follows:

(A) $a=1,2 \leq b, c \leq p-1 ; b=1,2 \leq a, c \leq p-1 ; c=1$, $2 \leq a, b \leq p-1$,

(B) $a=b=1,2 \leq c \leq p-1 ; a=c=1,2 \leq b \leq p-1$; $b=c=1,2 \leq a \leq p-1$,

(C) $a=b=c=1$.

In case (C), (14) becomes $d+e \equiv 2 \bmod p$ and $d^{2}+e^{2} \equiv$ $2 \bmod p$, so $d=e=1$. That is, in this case, (14) has only one solution $(a, b, c, d, e)=(1,1,1,1,1)$.

In case (B), if $a=b=1$ and $2 \leq c \leq p-1$, then (14) becomes $2 \leq c \leq p-1, d+e \equiv c+1 \bmod p, d^{2}+e^{2} \equiv c^{2}+$ $1 \bmod p$, and $d e \equiv c \bmod p$ or $(d-1)(e-1) \equiv 0 \bmod p$ and $\left(d^{2}-1\right)\left(e^{2}-1\right) \equiv 0 \bmod p, d e \equiv c \bmod p$ with $2 \leq c \leq p-1$. In this case, the number of the solutions of the congruence equation is $2(p-2)$. So, in case (B), the number of all solutions of congruence equation (14) is $3 \times 2(p-2)=6(p-2)$.

It is clear that the number of the solutions of congruence equation (14) in case (A) is three times the number of the solutions of the congruence equation $d+e \equiv b+c \bmod p$, $d^{2}+e^{2} \equiv b^{2}+c^{2} \bmod p$ with $2 \leq b, c \leq p-1$ and $1 \leq d, e \leq p-$ 1. While the number of the solutions of the latter congruence equation is $(p-1)(2 p-3)-4(p-2)-1$. In fact, the congruence equation $d+e \equiv b+c \bmod p, d^{2}+e^{2} \equiv b^{2}+c^{2} \bmod p$ with $1 \leq b, c, d, e \leq p-1$ is equivalent to congruence equation $d c+e c \equiv b c+c \bmod p, d^{2} c^{2}+e^{2} c^{2} \equiv b^{2} c^{2}+c^{2} \bmod p$, $1 \leq b, c, d, e \leq p-1$. So, from (B), we know that the number of the solutions is $(p-1)(2 p-3)$. From (B) and (C), we know that the number of the solutions of congruence equation (14) in case $(A)$ is $3 \times[(p-1)(2 p-3)-4(p-2)-1]$.
Combining three cases (A), (B), and (C), we deduce that the number of all solutions of (14) is

$$
\begin{aligned}
3 \times & {[(p-1)(2 p-3)-4(p-2)-1] } \\
& +6(p-2)+1=6 p^{2}-21 p+19
\end{aligned}
$$

Note that for all integers $1 \leq a, b, c, d, e \leq p-1$, the number of all solutions of the congruence equation

$$
\begin{aligned}
a+b+c & \equiv d+e+1 \bmod p, \\
a^{2}+b^{2}+c^{2} & \equiv d^{2}+e^{2}+1 \bmod p, \\
a^{k}+b^{k}+c^{k} & \equiv d^{k}+e^{k}+1 \bmod p, \\
a b c & \equiv d e \bmod p
\end{aligned}
$$

is also $6 p^{2}-21 p+19$. In fact, all solutions of (16) are the solutions of (11). Thus, they are also the solutions of (14). On the other hand, any solution in (14) must belong to case (A), (B), or (C). From the computational process of the solutions in these three cases, we can see that any solution must satisfy (16). So, the number of the solutions of congruence equation (16) is $6 p^{2}-21 p+19$.

Now, from (10), (11), (14), (15), and (16), we may immediately deduce the identity

$$
\begin{gathered}
\sum_{m=1}^{p} \sum_{n=1}^{p} \sum_{\chi \bmod p}\left|\sum_{a=1}^{p-1} \chi(a) e\left(\frac{a^{k}+m a^{2}+n a}{p}\right)\right|^{6} \\
=p^{2} \cdot(p-1)^{2} \cdot\left(6 p^{2}-21 p+19\right) .
\end{gathered}
$$

This completes the proof of our theorem.

\section{Conflict of Interests}

The authors declare that there is no conflict of interests regarding the publication of this paper.

\section{Acknowledgments}

The authors would like to thank the referees for their very helpful and detailed comments, which have significantly improved the presentation of this paper. This work is supported by the P.S.F. (2013JZ001) and N.S.F. (11371291) of China.

\section{References}

[1] T. M. Apostol, "An extension of the Lehmers' picturesque exponential sums," Mathematics of Computation, vol. 61, no. 203, pp. 25-28, 1993.

[2] B. C. Berndt, "On Gaussian sums and other exponential sums with periodic coefficients," Duke Mathematical Journal, vol. 40, pp. 145-156, 1973.

[3] T. Cochrane and C. Pinner, "Using Stepanov's method for exponential sums involving rational functions," Journal of Number Theory, vol. 116, no. 2, pp. 270-292, 2006. 
[4] T. Cochrane and Z. Zheng, "Upper bounds on a two-term exponential sum," Science in China A: Mathematics, vol. 44, no. 8, pp. 1003-1015, 2001.

[5] X. Du and D. Han, "On the fourth power mean of the three term exponential sums," Journal of Northwest University, vol. 43, pp. 541-544, 2013.

[6] L. K. Hua, Additive Prime Number Theory, Science Press, Beijing, China, 1957.

[7] W. M. Schmidt, Equations over Finite Fields: An Elementary Approach, vol. 536 of Lecture Notes in Mathematics, Springer, New York, NY, USA, 1976.

[8] A. Weil, "On some exponential sums," Proceedings of the National Academy of Sciences of the United States of America, vol. 34, pp. 204-207, 1948.

[9] W. Zhang and D. Han, "On the sixth power mean of the twoterm exponential sums," Journal of Number Theory, vol. 136, pp. 403-413, 2014.

[10] T. M. Apostol, Introduction to Analytic Number Theory, Springer, New York, NY, USA, 1976. 


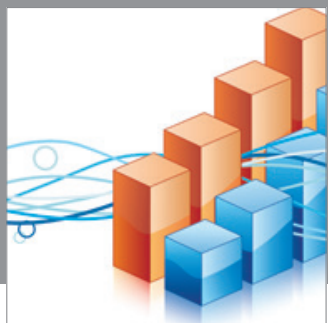

Advances in

Operations Research

mansans

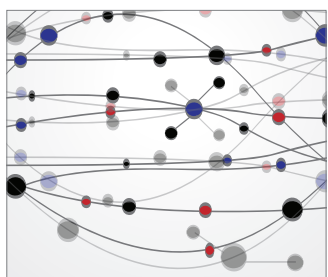

The Scientific World Journal
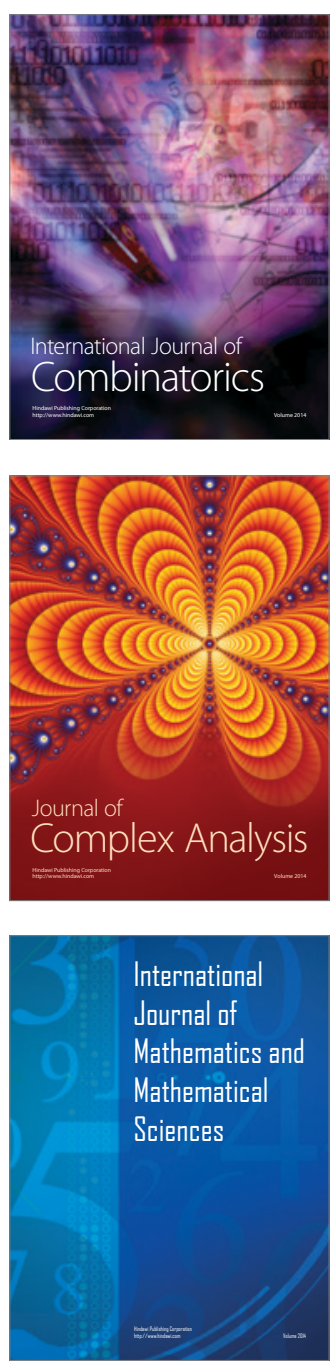
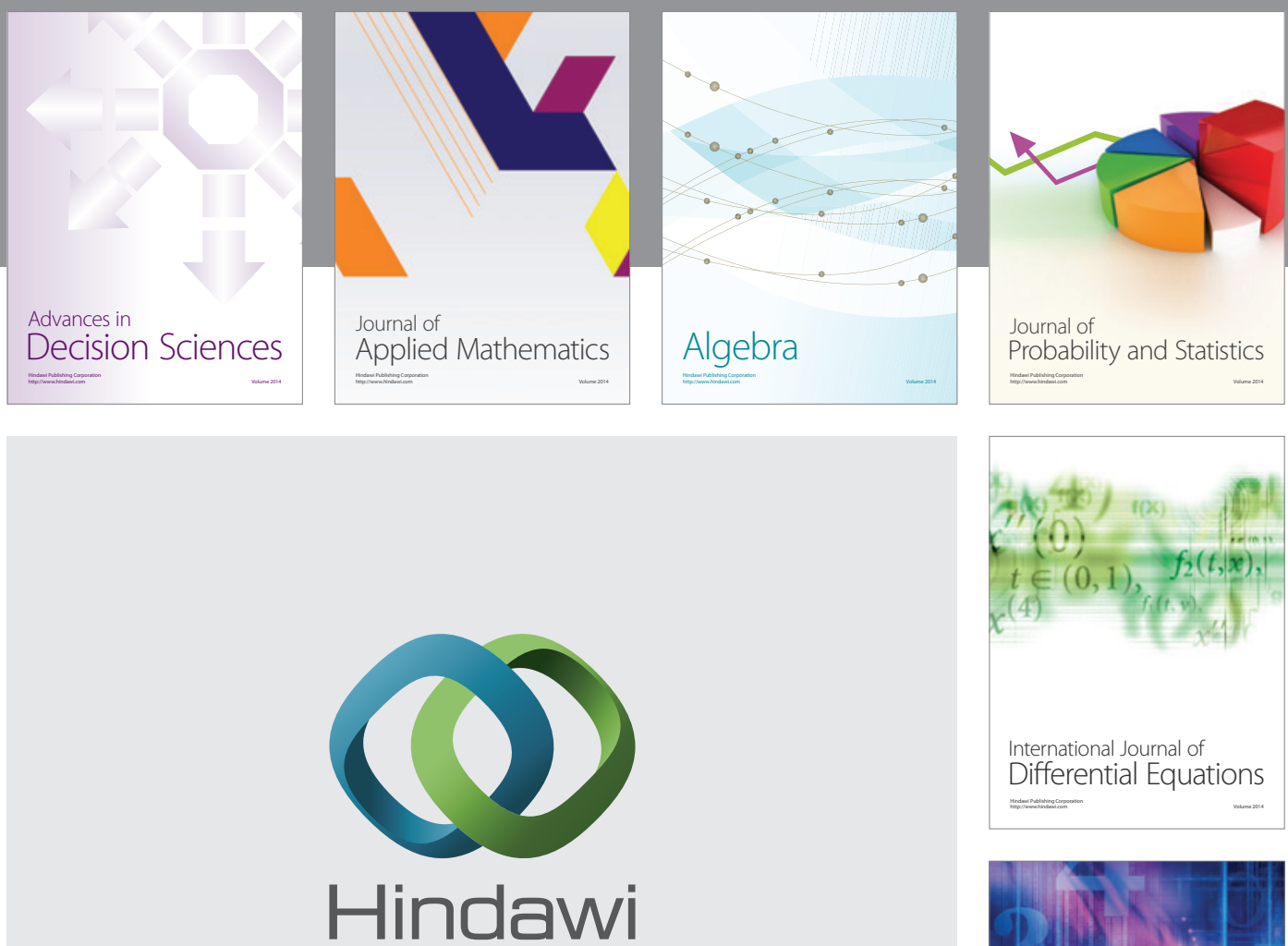

Submit your manuscripts at http://www.hindawi.com
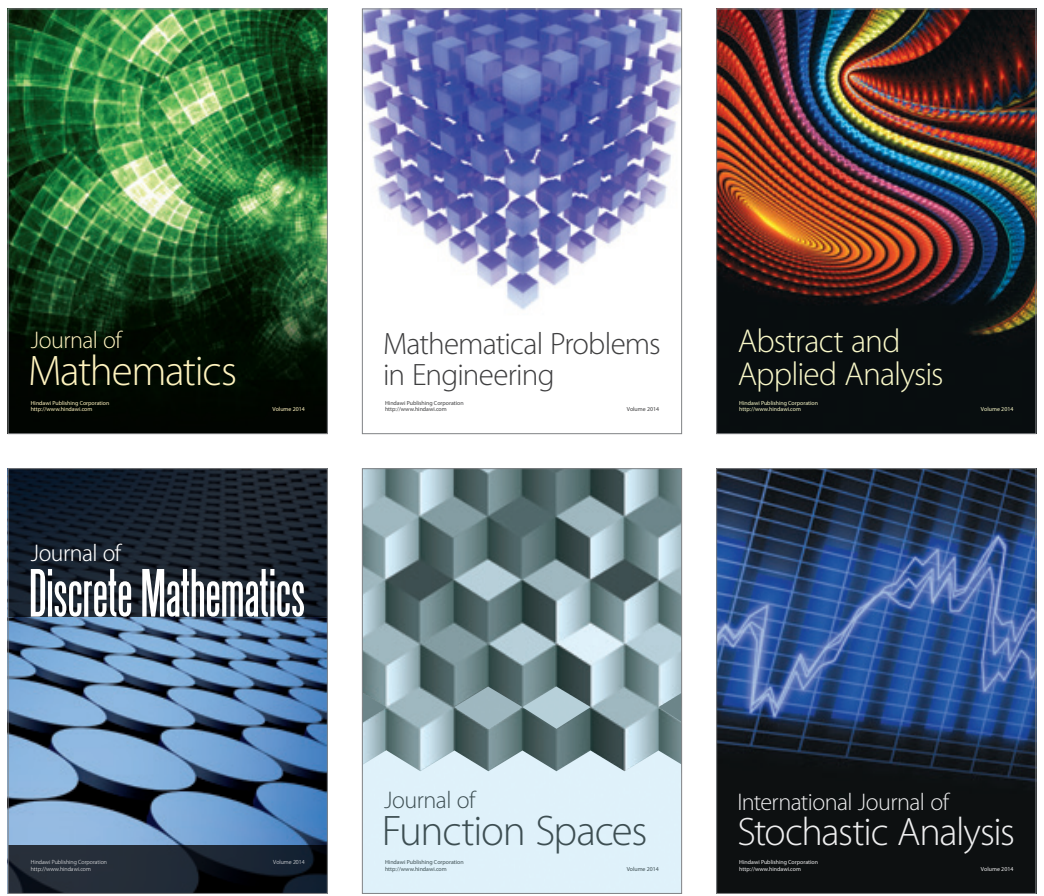

Journal of

Function Spaces

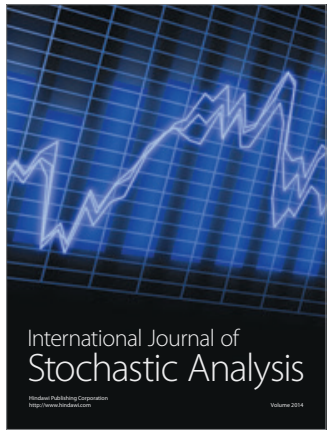

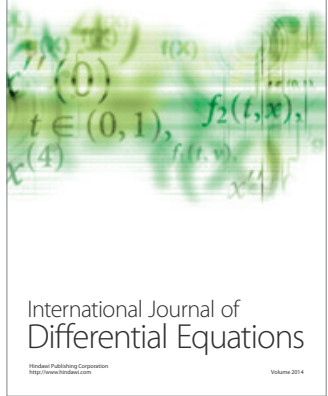
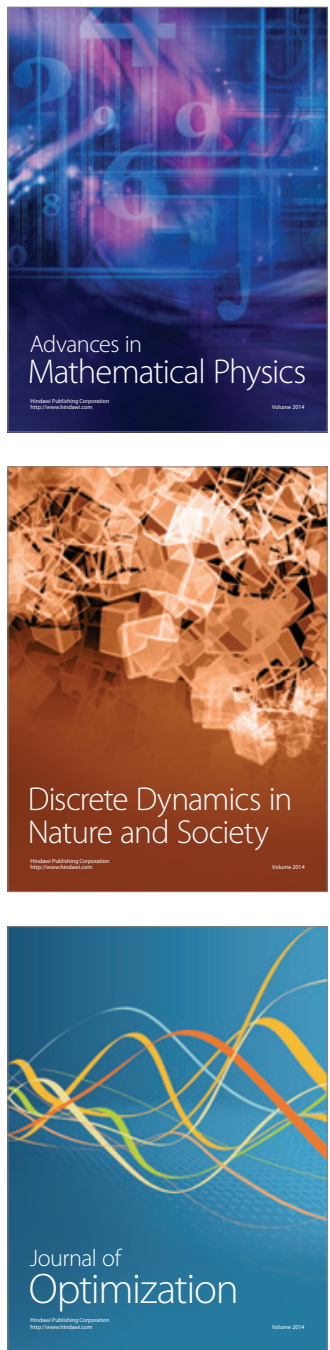